\title{
Isolated sub-cutaneous abcess of the buttock: An exceptional feature of tuberculosis
}

\section{Fatima Zahra Elfatoiki ${ }^{1}$, Maha Mouradi ${ }^{1}$, Hayat Dahbi Skali ${ }^{1}$, Fouzia Hali², Farida Marnissi', Soumia Chiheb1}

${ }^{1}$ Department of Dermatology, Ibn Rochd UHC, 1 quartier des Hopitaux 20000, Casablanca, Morocco, ${ }^{2}$ Department of Anatomo-Pathology, Ibn Rochd UHC, 1 quartier des Hopitaux 20000, Casablanca, Morocco

Corresponding author: Dr. Fatima Zahra Elfatoiki, E-mail: Fatiza59@hotmail.fr

\begin{abstract}
32-year-old patient, consulted for a tumefaction of the right buttock appeared 2 months ago associated with a fever and night sweats. Magnetic resonance imaging revealed an abscess. The tumefaction was drained. The pus culture showed Mycobacterium tuberculosis hominis. The patient was treated with a triple antibacillary therapy of 2 months (isoniazid, rifampicin, pyrazinamide) followed by dual therapy (isoniazid and rifampicin) for a total of 6 months with a good evolution.
\end{abstract}

Key words: Subcutaneous tuberculosis; Granuloma; Mycobacterium tuberculosis hominis 


\title{
Abcès sous cutané isolé: Une forme exceptionnelle de la tuberculose
}

\author{
Fatima Zahra Elfatoiki ${ }^{1}$, Maha Mouradi', Hayat Dahbi Skali ${ }^{1}$, Fouzia Hali², \\ Farida Marnissi', Soumia Chiheb1
}

${ }^{1}$ Department of Dermatology, Ibn Rochd UHC, 1 quartier des Hopitaux 20000, Casablanca, Morocco, ${ }^{2}$ Department of Anatomo-Pathology, Ibn Rochd UHC, 1 quartier des Hopitaux 20000, Casablanca, Morocco

Corresponding author: Dr. Fatima Zahra Elfatoiki, E-mail: Fatiza59@hotmail.fr

\begin{abstract}
RÉSUMÉ
Patiente de 32 ans, a consulté pour une tuméfaction de la fesse droite indolore, apparue 2 mois auparavant dans un contexte de fièvre non chiffrée et de sueurs nocturnes. Le reste de l'examen clinique était sans anomalies. L'IRM de la masse était en faveur d'un abcès. La masse était drainée. La culture du pus de drainage sur milieu de Lowenstein avait montré un Mycobacterium tuberculosis hominis. La patiente était traitée par une trithérapie antibacillaire de 2 mois (isoniazide, rifampicine, pyrazinamide) suivie d'une bithérapie (isoniazide et rifampicine) pour une durée totale de 6 mois avec une bonne évolution.
\end{abstract}

Mots clé: Tuberculose sous cutanée; Granulome; Mycobacterium tuberculosis hominis

\section{INTRODUCTION}

La tuberculose sous cutanée de type abcès froid est une forme rare de tuberculose survenant le plus souvent dans un contexte de tuberculose grave et disséminée ou compliquant une tuberculose pleuropulmonaire ou ostéoarticulaire. Nous rapportons le cas d'un abcès froid sous cutané isolé chez une jeune patiente.

\section{OBSERVATION}

Patiente de 32 ans, marocaine, sans antécédents pathologiques particuliers, ni contage tuberculeux, vaccinée par le BCG, a consulté pour une tuméfaction de la fesse droite indolore, apparue 2 mois auparavant dans un contexte de fièvre non chiffrée et de sueurs nocturnes sans amaigrissement associé. À l'examen, elle était en bon état général, apyrétique. On constatait une tuméfaction de la partie supérieure de la fesse droite, mesurant $10 \mathrm{~cm}$ du grand axe, molle, indolore, avec une peau d'aspect normal en regard (Fig. 1). Lauscultation pulmonaire était normale. Lexamen du rachis était normal, sans raideur, ni impotence fonctionnelle ni douleur provoquée. Le reste de l'examen clinique était sans anomalies.

L'IRM de la masse était en faveur d'un abcès. La radiographie du thorax et le scanner du rachis dorsolombaire étaient sans anomalies. Lintradermoréaction à la tuberculine était phlycténulaire et l'examen cytobactériologique des expectorations était négatif.

La masse était drainée (Fig. 2) avec réalisation de prélèvements bactériologiques du pus et d'une biopsie de la coque de l'abcès. La culture du pus de drainage sur milieu de Lowenstein avait montré un Mycobacterium tuberculosis hominis et la biopsie de coque était en faveur d'un granulome épithéliogigantocellulaire sans nécrose caséeuse (Fig. 3), la coloration de Ziehl était négative.

La patiente était traitée par une chimiothérapie antituberculeuse selon le protocole de l'OMS avec une trithérapie antibacillaire de 2 mois (isoniazide, rifampicine, pyrazinamide) suivie d'une bithérapie

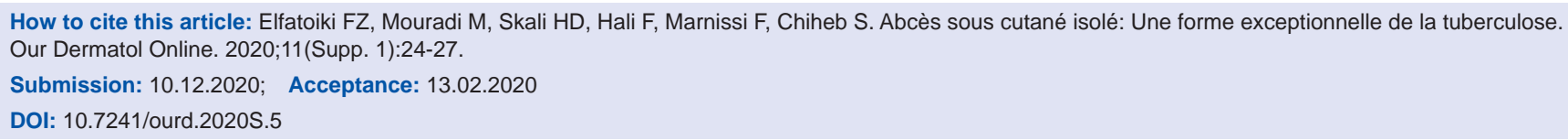




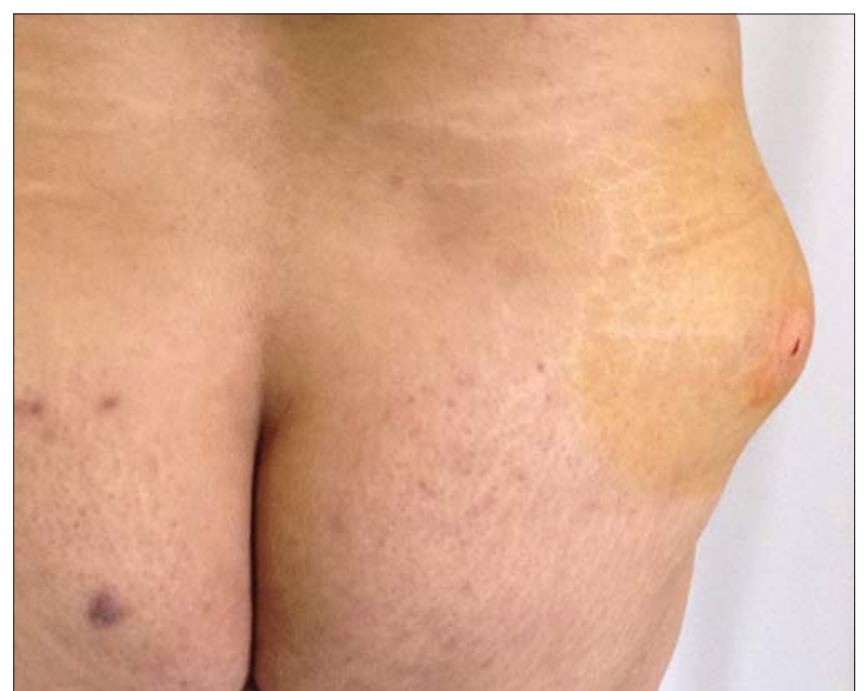

Figura 1: Tuméfaction de la partie supérieure de la fesse droite.

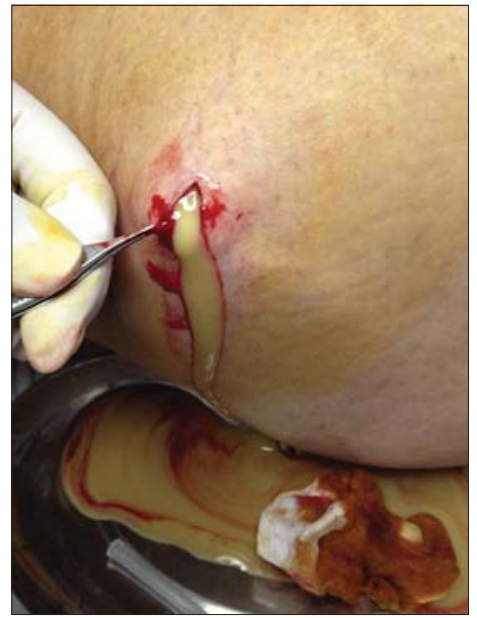

Figura 2: Drainage de l'abcès froid.

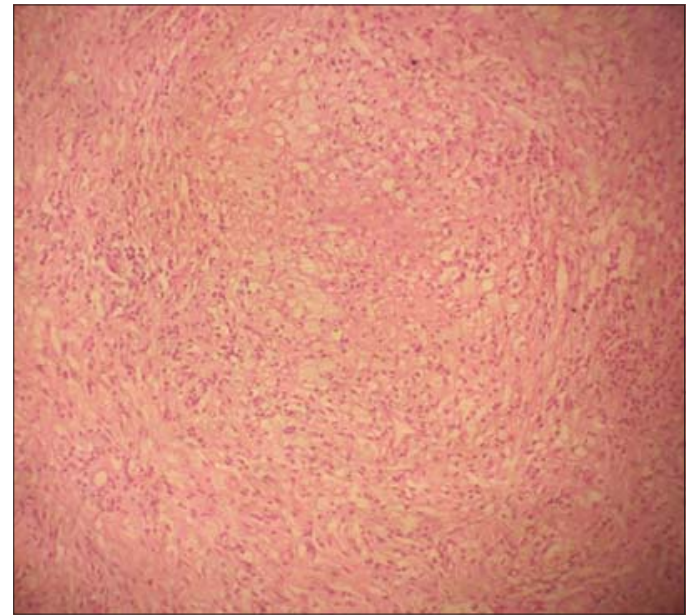

Figura 3: Granulome épithéliogigantocellulaire sans nécrose caséeuse.

(isoniazide et rifampicine) pour une durée totale de 6 mois.
L'évolution était marquée par une régression des signes généraux et un assèchement de l'abcès de la fesse après 2 mois de traitement. Il n'y avait pas eu de récidive de la masse à l'arrêt de la chimiothérapie antituberculeuse. Le recul actuel est de 8 mois.

\section{DISCUSSION}

Loriginalité de notre observation réside dans la rareté de l'abcès froid sous cutanés au cours de la tuberculose et sa survenue isolée chez notre patiente en dehors de toute atteinte ostéoarticulaire.

Les abcès froids sous cutanés au cours de la tuberculose sont exceptionnels. La localisation pariétale thoracique est la plus fréquemment observé et représente moins de $5 \%$ des atteintes tuberculeuses ostéoarticulaires [1]. Elle pose un problème de diagnostique avec les tumeurs pariétales et avec les autres infections pariétales [1,2]. Cette forme de tuberculose peut être isolée ou secondaire à une atteinte pleuropulmonaire $[3,4]$. Cette localisation peut être secondaire à une dissémination hématogène ou lymphatique à partir d'un foyer pleuropulmonaire, à une inoculation directe transcutanée ou à une extension par contiguité à partir d'une adénite de la paroi thoracique ou d'un empyème tuberculeux $[5,6]$. Labcès froid peut se fistuliser à la peau ou diffuser à distance et donner une localisation secondaire [6]. Lobservation de Mercier et al illustre une spondylodiscite L4-L5 compliquée d'un abcès du psoas et d'un abcès périvertébral avec une fistulisation vers les tissus mous paravertébraux à gauche et au niveau de la fesse gauche [7].

Dans notre cas, la localisation au niveau de la fesse est survenue en dehors de toute atteinte ostéoarticulaire ou pulmonaire et pourrait être due à une inoculation directe transcutanée par le bacille de Koch.

\section{CONCLUSION}

La tuberculose cutanée est toujours une affection d'actualité dans les pays en voie de développement tel le Maroc. Son polymorphisme clinique peut parfois être responsable d'un retard diagnostique.

\section{Consent}

The examination of the patient was conducted according to the Declaration of Helsinki principles. 


\section{www.odermatol.com}

The authors certify that they have obtained all appropriate patient consent forms. In the form the patient(s) has/have given his/her/ their consent for his/her/their images and other clinical information to be reported in the journal. The patients understand that their names and initials will not be published and due efforts will be made to conceal their identity, but anonymity cannot be guaranteed.

\section{RÉFÉRENCES}

1. Mu J, Liu ZC, Zhang C, Wang CL, Zhang HQ. Zhonghua Bing Li Xue Za Zhi. 2020;49:562-7.

2. Hussain M, Yang A, Yassin M, Arbulu R, Phan T. MALDITOF vs. VITEK 2 for identification of Aggregatibacter actinomycetemcomitans chest wall abscess. IDCases. 2020;20:e00749.

3. Motoki S, Yuzo S, Hikotaro K. Surgical treatment of tuberculous abscess in the chest wall. Ann Thorac Surg. 2005;79:964-7.

4. Fenniche S, Fekih L, Hassene H, Hantous S, Belhabib D,
Megdiche ML. Tuberculous thoracic parietal abcess. Report of 4 cases. Tunis Med. 2003;81:42.

5. Trombati N, Afif H, Farouki El Z, Bahlaoui A, Aichane A, Bouayad Z. La tuberculose pariétale thoracique en dehors de l'immunodépression par le virus de l'immunodéficience humaine. Rev Mal Respir. 2001;18:301-4.

6. Mahouachi R, Zendah I, Taktak S, Chtourou A, Ben Chaabane R, Gharbi R. Tuberculose de la paroi thoracique. Rev Pneumol Clin. 2006;62:56-8.

7. Mercier A, Pouessel G, Dos Remedios J, Dewatre F, GangaZandzoua S, Ythier H. Abcès de la fesse et toux chronique chez une adolescente. Archives de pédiatrie. 2007;14:73-6.

Copyright by Fatima Zahra Elfatoiki, et al. This is an open-access article distributed under the terms of the Creative Commons Attribution License which permits unrestricted use, distribution, and reproduction in any medium, provided the original author and source are credited.

Source of Support: Nil, Conflict of Interest: None declared. 